Polymer Journal, Vol. 39, No. 4, pp. 389-396 (2007)

(C) 2007 The Society of Polymer Science, Japan

\title{
High Modulus and High Strength Fibers with High Electric Conductivity Prepared by Copper Electroless Plating on the Surface of Poly ( $p$-phenylenebenzobisoxazole) (PBO)
}

\author{
Masaru MATSUO ${ }^{\dagger}$, Hisako IsHIKAwA, Ying XI, and Yuezhen BiN \\ Department of Textile and Apparel Science, Faculty of Human Life and Environment, \\ Nara Women's University, Nara 630-8263 Japan
}

(Received November 15, 2006; Accepted January 24, 2007; Published March 12, 2007)

\begin{abstract}
In attempt to produce high modulus and high strength fibers with high electric conductivity, copper plating on the surface of poly ( $p$-phenylenebenzobisoxazole) (PBO) fiber was carried out by using electroless plating, since PBO is a super-heat resistant polymer with the highest Young's modulus among polymers but is an insulator. The mechanical and electrical properties of the plated fibers were investigated as a function of copper content. At the maximum volume fraction of copper, $c a .30 .1 \mathrm{vol} \%$, the electric conductivity reached $10^{5.1} \mathrm{~S} / \mathrm{cm}$ and the corresponding Young's modulus was $c a$. $130 \mathrm{GPa}$. The stress distribution in the radial direction within the plated fiber was calculated on the basis of a continuous mechanical theory, when external stress was applied along the plated fiber axis. The calculated stress gap at the boundary between PBO and copper phases became larger with increasing copper content. However, the peeling phenomenon at the boundary did not occur under the elongation up to breaking, indicating that the adhesion between the copper and PBO phases is enough to conquer the stress concentration at the boundary under the external applied excitation. [doi:10.1295/polymj.PJ2006161]

KEY WORDS Poly( $p$-phenylenebenzobisoxazole) (PBO) / Copper Electroless Plating / Mechanical Properties / Electrical Properties /
\end{abstract}

Poly ( $p$-phenylenebenzobisoxazole) (PBO) fiber, which is well known as Zylon, is a super heat resistant polymer with the highest Young's modulus among all commercial fibers at present. ${ }^{1,2}$ The value of Young's modulus is higher than that of steel. Thus, PBO shows the most attractive properties and the greatest economic potentiality among the polybenzazoles. Especially, because of its light weight associated with essential characteristics of polymers, the further utility of PBO fibers can be expected in many fields such as aircraft and aerospace, and then a number of papers about PBO fibers have been reported in terms of the fundamental aspects. ${ }^{3-7}$

The plating of metals on the fibers has been investigated mainly for carbon fibers with the aim of completely isolating the fiber from its surrounding. ${ }^{8-10}$ The main focus of these investigations was concentrated on the improvement of interfacial properties between fibers and epoxy matrix in a composite system.

Apart from their focus, it is also of interest to prepare high conductive fibers with the highest modulus and highest strength by plating copper on the surface of PBO fiber, since the PBO fibers have much better mechanical properties and heat-resistance than Kevlar fibers. To achieve high electric conductivity similar to metals, large amounts of metals with very high conductivity such as copper must be plated on the surface of PBO fibers. This paper deals with the trial for plat- ing PBO fibers with metals.

Here it should be noted that as one of the characteristics of metals, most of metals transform from solid state to liquid state beyond their melting point and their mechanical strength becomes zero immediately. On the other hand, PBO fibers start to pyrolyze at ca. $600^{\circ} \mathrm{C}$ but any drastic disruption does not occur in a moment even when PBO fibers are put in fire. ${ }^{11}$ Furthermore, the copper-plated PBO fiber can recover from large deformation by bending immediately, while most of metal wires take plastic deformation against bending. Such high elasticity of PBO fibers also provides the greatest potentiality as a new kind of electric wires. The main focus of this paper is concentrated on the preparation and the characteristics for copper-plated PBO fibers by electroless plating methods.

\section{EXPERIMENTAL}

\section{Sample Preparation}

PBO fibers used in the present experiment have the characteristics with filament decitex $(1.7 \mathrm{dtex})$, density $\left(1.56 \mathrm{~g} / \mathrm{cm}^{3}\right)$, tensile strength $(5.8 \mathrm{GPa})$, Young's modulus (270 GPa), elongation at break $(2.5 \%)$ and moisture regain $(0.6 \%)$. They were furnished from Toyobo Co. Ltd. Chart 1 shows the process for electroless plating of PBO fibers.

${ }^{\dagger}$ To whom correspondence should be addressed (Tel/Fax: +81-742-20-3462, E-mail: m-matsuo@cc.nara-wu.ac.jp). 


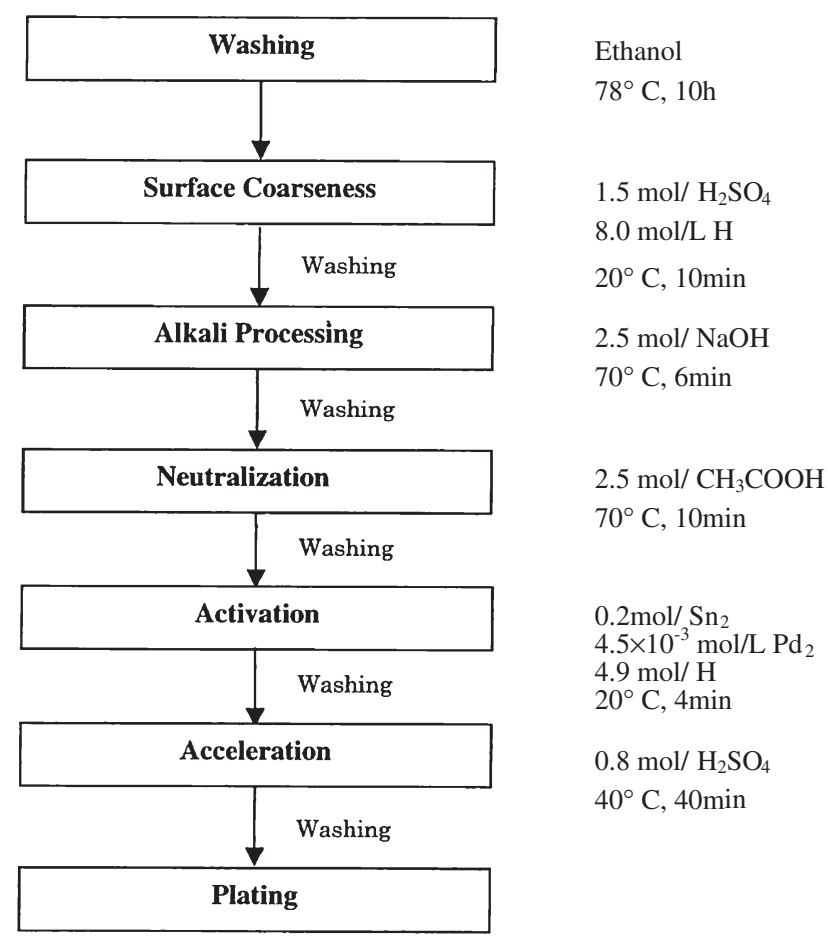

Chart 1. Processes for electroless plating of PBO fibers.

As listed in Chart 1, first of all, the surface of PBO fibers with $7 \mathrm{~cm}$ length were washed by ethanol at $78^{\circ} \mathrm{C}$ for $10 \mathrm{~h}$ and dried at room temperature. The solution containing $8.0 \mathrm{~mol} / \mathrm{L}$ of $\mathrm{HCl}$ and $1.5 \mathrm{~mol} / \mathrm{L}$ of $\mathrm{H}_{2} \mathrm{SO}_{4}$, was prepared and the PBO fibers were immersed in the $30 \mathrm{~mL}$ solution for $10 \mathrm{~min}$ at $20^{\circ} \mathrm{C}$ to give coarseness on their surface by etching. After washing, the fibers were immersed in $30 \mathrm{~mL} \mathrm{NaOH}$ solution with $2.5 \mathrm{~mol} / \mathrm{L}$ concentration for $6 \mathrm{~min}$ at $70^{\circ} \mathrm{C}$, as alkali processing. After washing, the fibers were immersed in $30 \mathrm{~mL} \mathrm{CH}_{3} \mathrm{COOH}$ with $2.5 \mathrm{~mol} / \mathrm{L}$ concentration for $6 \mathrm{~min}$ at $70^{\circ} \mathrm{C}$ for neutralization and then washed with millipore water. $4.5 \times 10^{-3}$ $\mathrm{mol} / \mathrm{L}$ of $\mathrm{PdCl}_{2}, 0.2 \mathrm{~mol} / \mathrm{L}$ of $\mathrm{SnCl}_{2}$ and $4.9 \mathrm{~mol} / \mathrm{L}$ of $\mathrm{HCl}$ were stirred together to prepare the activation solution whose volume is $20 \mathrm{~mL}$. The fibers were stirred in the activation solution for $4 \mathrm{~min}$ at $20^{\circ} \mathrm{C}$. The activated fibers were picked up from the activation medium and washed with millipore water. The activated fibers were stirred in $30 \mathrm{~mL}$ aqueous solution with the concentration of $0.8 \mathrm{~mol} / \mathrm{L} \mathrm{H}_{2} \mathrm{SO}_{4}$ for $4 \mathrm{~min}$ at $40^{\circ} \mathrm{C}$ to pursue acceleration and then washed with millipore water. After drying, the weight of the PBO fibers was measured. The surface treatment shown in Chart 1 is thought to be the best condition to carry out copper plating on PBO fibers. The weight loss by the chemical modification was so small that it can be negligible. The several isolated fibers after the chemical modification on their surfaces were put into a bath with chemical composition listed in Table I to pursue copper plating on their surface. The immersion
Table I. Chemical components of electroless copper plating

\begin{tabular}{lc}
\hline \multicolumn{1}{c}{ Chemicals } & $\begin{array}{c}\text { Concentration } \\
(\mathrm{mol} / \mathrm{L})\end{array}$ \\
\hline $\mathrm{CuSO}_{4} \cdot 5 \mathrm{H}_{2} \mathrm{O}$ & 0.04 \\
$4 \mathrm{Na}-\mathrm{salt}_{\mathrm{s}}$ of ethylenediamine- & 0.12 \\
tetraacetic acid (EDTA) & 0.20 \\
$\mathrm{HCHO}$ & 0.14 \\
$\mathrm{Na}_{2} \mathrm{SO}_{4}$ & 0.30 \\
$\mathrm{HCOONa}$ & $4.6 \times 10^{-4}$ \\
$\mathrm{KCN}$ & \\
\hline
\end{tabular}

time was selected to control the plating amount of copper. The resultant copper-plated fibers were washed with millipore water. After drying, the weight of the copper-plated PBO fibers was measured to calculate volume fraction of plated copper.

\section{Experimental Procedure}

The Young's modulus and tensile strength for the plated PBO fibers, the length to be drawn being $20 \mathrm{~mm}$, were measured by a testing instrument, TENSILON/STM-H-500BP of Orientic Co. Ltd., at room temperature and at cross head speed of $2 \mathrm{~mm} /$ min. The bundle of the fibers after the chemical modification was used as a test specimen, since the detected stress by elongation of a single PBO fiber is too small to obtain the accurate data. The cross-section area of a PBO fiber was calculated as the average value of the test PBO fibers by assuming that the cross section is circular. In doing so, the weight of the bundle, the number of the fiber in a bundle, fiber length and density were measured carefully for the calculation of the cross section. The cross-section area of the copper-plated PBO fibers was calculated by adding the cross-section area of plated copper layer obtained from weight of the plated copper, fiber length and copper density.

The electric conductivity was measured by using a four-terminal method at room temperature by using H2-3000 of Hokuto Electric Co. Ltd.

Scanning electron microscopy (SEM) and Energy Dispersion X-ray Spectrometry (EDS) were obtained with a JSM-T300 of JEOL Co. Ltd. The observation was possible for the surface but impossible for the cross-section because of the difficulty in cutting and breaking of the plated fibers without deformation of their circular cross-section in spite of a lot of efforts.

$\mathrm{X}$-Ray diffraction intensity distributions were measured by a $12 \mathrm{~kW}$ rotating-anode $\mathrm{X}$-ray generator (Rigaku RAD-rA) with the monochromatic $\mathrm{CuK} \alpha$ radiation (wavelength of $0.1542 \mathrm{~nm}$ ). The measurement by a reflection method was done from $5^{\circ}$ to $60^{\circ}$ (twice the Bragg angle) with a step-scanning device at a step interval of $0.1^{\circ}$, each fixed time of $40 \mathrm{~s}$. 


\section{RESULTS AND DISCUSSION}

Figure 1 shows the volume fraction (vol \%) of copper against a copper-plated PBO fiber as a function of immersing time in the plating bath. It is seen that the plating content tends to level off drastically beyond $60 \mathrm{~min}$, although the results are not shown in this paper. The plots show the average value at each plating time. The maximum volume fraction of plated cupper was $c a .30 .1 \%$ indicating the limit of the planting power of the bath. The volume fraction $\varphi_{V}$ can be evaluated by using weight fractions of copper $\left(\varphi_{W}\right)$, density of a PBO fiber $\left(\rho_{\text {PBO }}=1.56 \mathrm{~g} / \mathrm{cm}^{3}\right)$ and density of copper $\left(\rho_{\mathrm{Cu}}=8.95 \mathrm{~g} / \mathrm{cm}^{3}\right)$ as follows:

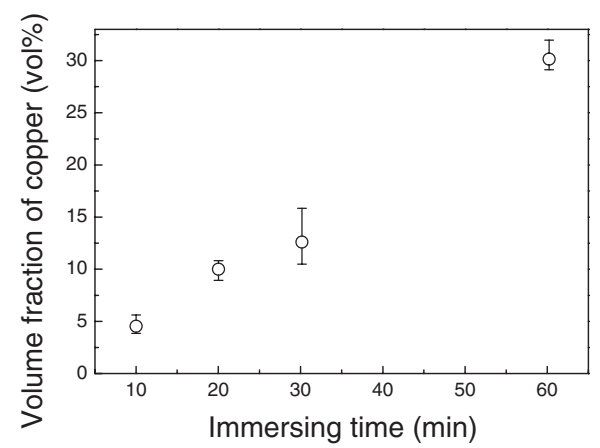

Figure 1. Volume fraction (vol\%) of copper plated on the PBO fibers as a function of immersing time in the plating bath.
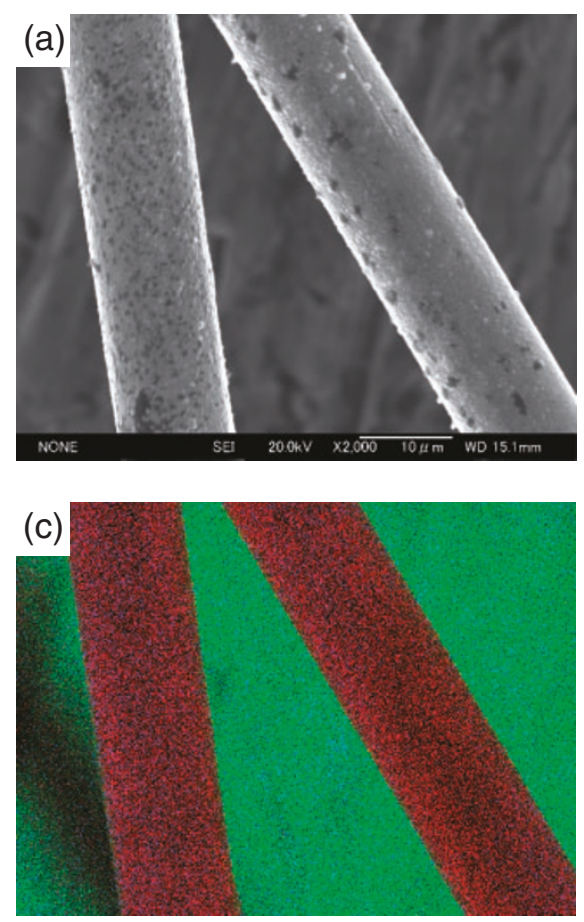

$$
\varphi_{V}=\frac{\rho_{\mathrm{PBO}} \varphi_{W}}{\varphi_{W} \rho_{\mathrm{PBO}}+\rho_{\mathrm{Cu}}\left(1-\varphi_{W}\right)}
$$

In eq 1 , the weight fraction of copper $\left(\varphi_{W}\right)$ can be obtained easily by the weight of an original PBO fiber and the weight of a copper plated PBO fiber.

Figure 2(a) and (b) show SEM photographs of the copper-plated fibers, which were taken on their surface. The corresponding EDS photographs in Figure 2(c) and (d) of the copper-plated fibers were also taken at the same place with SEM photograph. The red color is copper, and green color is aluminum (the sample table is made of aluminum). The SEM photos reveal that the number of copper particles covered on the surface of a PBO fiber increases as the plating time increases. Namely, the copper plating of fibers immersed in the bath for $60 \mathrm{~min}$ is thought to be thicker than that for $10 \mathrm{~min}$, since the surface seems to be covered uniformly by copper particles. On the other hand, the EDS photos reveal that the PBO fibers were covered by copper layer even at 10 min. However, the thickness of the copper layer is very important to prepare the fibers with high electric conductivity.

To check the plating condition, WAXD intensity distribution curves were obtained for original PBO fibers and plated fibers prepared by immersing PBO fibers for $60 \mathrm{~min}$ in the plating bath. To emphasize the diffractions from PBO crystallites, the fibers were arranged in the vertical direction to detect some sharp
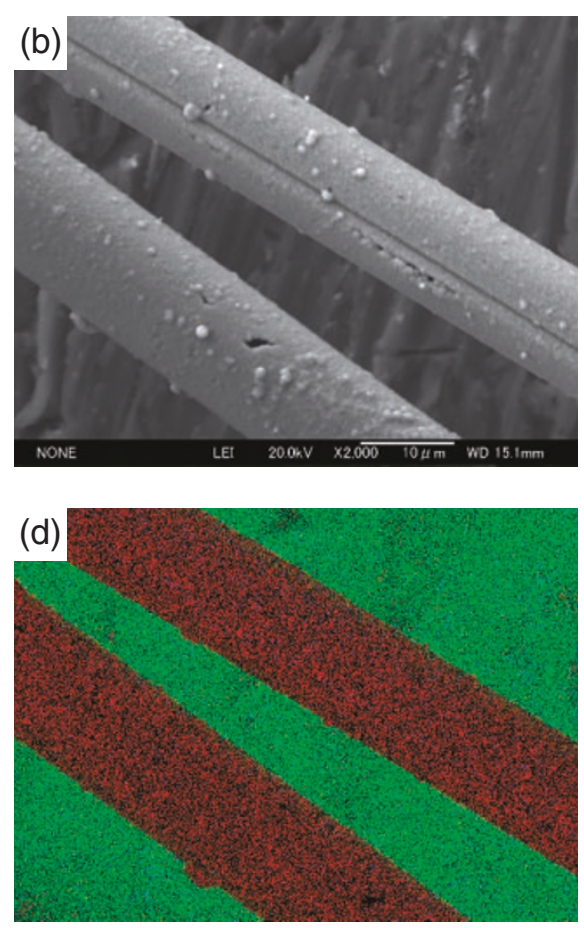

Figure 2. SEM photographs (a) and (c) and the corresponding EDS photos (b) and (d). The photos (a) and (c) were taken for the fibers plated for $10 \mathrm{~min}$ in a plating bath, while (b) and (d) for $60 \mathrm{~min}$. 


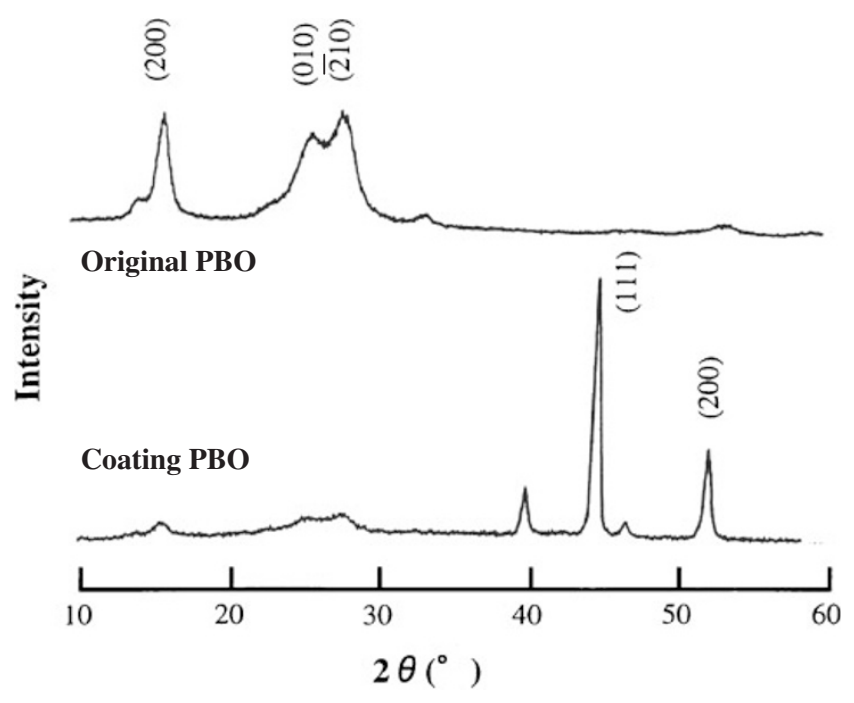

Figure 3. X-Ray diffraction intensity curves measured for the PBO fiber and copper-plated PBO fiber.

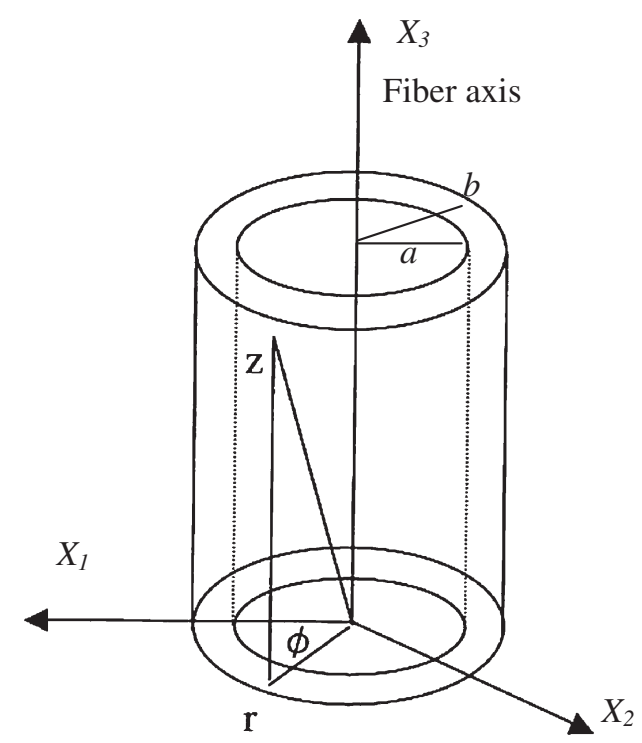

Figure 4. Cylindrical coordinates fixed within the plated PBO fiber.

spots on the equator and Figure 3 shows the results. As for PBO fibers, the peaks from the (200), (010) and (210) planes can be clearly observed. ${ }^{1}$ On the other hand, the very strong peaks from copper crystallites are observed for the plated fibers, but the very weak diffraction peaks from $\mathrm{PBO}$ crystallites are also observed indicating that the copper layer (ca. $30.1 \mathrm{vol} \%$ ) plated on the PBO fiber allows penetration of an incident X-ray beam by a $12 \mathrm{~kW}$ rotatinganode $\mathrm{X}$-ray generator.

Judging from Figures 2 and 3, a cylindrical model as shown in Figure 4 may be proposed, in which $a$ and $b$ are the radiuses of a PBO fiber and a copper plated fiber, respectively. In this model system, copper is plated with the uniform thickness, $b-a$, around a PBO fiber whose radius is $a$, and the third layer

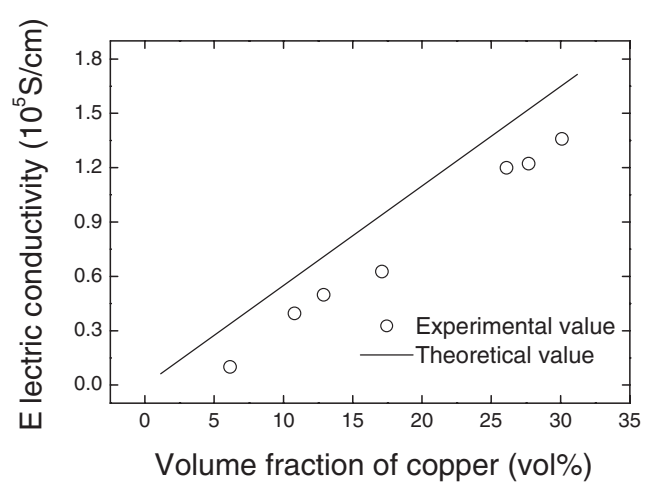

Figure 5. Electric conductivity of the plated fibers as a function of copper content.

formed at $\mathrm{PBO} / \mathrm{Cu}$ interface by the chemical modification of PBO surface is neglected. Actually, the mechanical constants and thickness of the third layer cannot be postulated.

Since the cylindrical model in Figure 4 belongs to a parallel model, the Young's modulus $E$ of the plated fiber is given by

$$
E=\left(1-\varphi_{V}\right) E_{\mathrm{PBO}}+\varphi_{V} E_{\mathrm{Cu}}
$$

where $E_{\mathrm{PBO}}$ and $E_{\mathrm{Cu}}$ are the measured Young's modulus of PBO fiber and copper, respectively. A volume fraction of copper $\varphi_{V}$ is calculated by using eq 1 .

Similarly, the electric conductivity $S$ of the plated fiber is also given by

$$
S=\left(1-\varphi_{V}\right) S_{\mathrm{PBO}}+\varphi_{V} S_{\mathrm{Cu}}
$$

where $S_{\mathrm{PBO}}$ and $S_{\mathrm{Cu}}$ are the electric conductivity of PBO fiber and copper, respectively.

Figure 5 shows average value for electric conductivity of the plated fibers as a function of volume fraction of copper, in which the solid line is the theoretical electric conductivity calculated by eq 3 . The experimental value of the electric conductivity is proportional to the copper volume fraction and the same tendency is also confirmed for the theoretical line. The experimental values are lower than that of the calculated values in the given volume fraction of copper. The reduction of the experimental value from the theoretical value is thought to be due to the impurities such as tin and palladium introduced by activation process before copper plating. The slight agreement between the experimental and the theoretical values also approves that the PBO fibers were covered by copper very well even at very small content ( $c a$. $6.14 \mathrm{vol} \%$ ). The value, $10^{5.1} \mathrm{~S} / \mathrm{cm}$, at $c a .30 .1 \mathrm{vol} \%$ is slightly lower than the conductivity of copper, $10^{5.7} \mathrm{~S} / \mathrm{cm}$, but this is almost equal to the conductivities of chromium and platinum.

Figure 6(a) and (b) show the plots of the average values for Young's modulus and tensile strength as 




Figure 6. (a) Young's modulus and (b) tensile strength as a function of copper content, in which line in (a) is the theoretical arithmetic results.

a function of copper content. By the etching, activation and acceleration processes as listed in Chart 1 in Experimental, the Young's modulus and tensile strength of the PBO fibers after chemical modification decrease in comparison with the reported Young's modulus $(270 \mathrm{GPa})$ and the tensile strength $(5.8 \mathrm{GPa})$ of the original fibers. ${ }^{1}$ The values of Young's modulus and tensile strength become lower with increasing copper content by the plating, while the corresponding electric conductivity increased as shown in Figure 5. The Young's moduli at $6.14 \mathrm{vol} \%$ and $30.1 \mathrm{vol} \%$ are $180 \mathrm{GPa}$ and $130 \mathrm{GPa}$, respectively. Even so, $130 \mathrm{GPa}$ is slightly higher than the Young's modulus $(110 \mathrm{GPa})$ of copper. This plated fiber is surely a high modulus and high strength fiber with high electric conductivity. The tensile strength of the plated fiber decreased with increasing copper content and became $2.5 \mathrm{GPa}$ at $30.1 \mathrm{vol} \%$ in Figure 6(b). Even so, this tensile strength is much higher than the value ( $c a$. $360 \mathrm{MPa}$ ) of original copper wire and also higher than the value $(\mathrm{ca} .700 \mathrm{MPa})$ of copper alloy wire $\left(\mathrm{CuNi}_{3}-\right.$ $\mathrm{SiMg}$ ) produced by Wieland-Werke AG company. Here we must emphasize that any peeling did not occur under elongation up to the breaking of the specimens. The solid line in Figure 6(a) is the theoretical results calculated by eq 2 . The calculated values are slightly higher than the experimental values. The slightly disagreement is due to the fact that although the calculation is carried out by using the average measured Young's modulus of PBO fibers whose surface was damaged by the etching, activation and acceleration processes, the decrease of the Young's modulus by immersing the PBO fibers in the plating bath is neglected. Unfortunately, there is no way how to estimate the decrease of Young's modulus by the plating process at present. Even so, we must emphasize that the derivation from a simple additive law is attributed to the decrease in Young's modulus of $\mathrm{PBO}$ fibers under plating process.

Another interesting phenomenon for the plated fiber is bending properties. The plated fiber could recover to a straight line immediately, even when it is bent perfectly. The rapid $180^{\circ}$ recovery is attributed to a high elasticity of PBO fibers because of the rigidrod like nature. Such property cannot be generally realized for metals with plastic deformation against bending.

Here a question arises as to whether the plated fiber can be used as electric wires. To justify the utility as electric wires, it is important to clarify the concentrated stress arisen at the boundary region between the PBO and copper phases theoretically, when an external excitation is applied to the plated fiber. The mathematical calculation by using a cylindrical coordinate was carried out for the stress distribution in a radial direction within a plated fiber. In this model system, when an external stress is applied along the fiber axis, the stress-strain relationship within a plated fiber may be described by the generalized Hook's law.

$$
\begin{aligned}
\sigma_{r r} & =C_{11} \varepsilon_{r r}+C_{12} \varepsilon_{\phi \phi}+C_{13} \varepsilon_{z z} \\
\sigma_{\phi \phi} & =C_{12} \varepsilon_{r r}+C_{12} \varepsilon_{\phi \phi}+C_{13} \varepsilon_{z z} \\
\sigma_{z z} & =C_{13} \varepsilon_{r r}+C_{13} \varepsilon_{\phi \phi}+C_{33} \varepsilon_{z z} \\
\sigma_{z r} & =2 C_{44} \varepsilon_{z r} \\
\sigma_{z \phi} & =\sigma_{r \phi}=0
\end{aligned}
$$


where $\sigma_{i j}$ and $\varepsilon_{i j}$ are local stress and strain within a fiber, respectively and $C_{i j}$ are elastic stiffness. The values $C_{i j}$ in eq 4 are inverse relationship with $S_{i j}$ and they are obtained by using the following equation.

$$
\begin{gathered}
\Delta_{i j}=(-1)^{i+j} \Delta_{i j}^{s} / \Delta^{s} \\
\left|\begin{array}{cccccc}
S_{11} & S_{12} & S_{13} & 0 & 0 & 0 \\
S_{12} & S_{11} & S_{13} & 0 & 0 & 0 \\
S_{13} & S_{13} & S_{33} & 0 & 0 & 0 \\
0 & 0 & 0 & S_{44} & 0 & 0 \\
0 & 0 & 0 & 0 & S_{44} & 0 \\
0 & 0 & 0 & 0 & 0 & S_{66}
\end{array}\right|
\end{gathered}
$$

where $\Delta_{i j}^{s}$ is the determinant of the matrix obtained by omitting the $i$-th line and $j$-th rank in the matrix $S_{i j}$ and $\Delta^{s}$ is the determinant of matrix $S_{i j}$.

To obtain the values of $C_{i j}$, the relationship between the intrinsic compliance of the structural unit and the bulk compliance is given as angular relationship in Figure 7. Figure 7 shows a Cartesian coordinate $O$ $U_{1} U_{2} U_{3}$ fixed within a crystal unit as a structural unit, with respect to another Cartesian coordinate $O$ -
$X_{1} X_{2} X_{3}$ fixed in a bulk specimen. The $X_{3}$ and $U_{3}$ axis may be taken along the PBO fiber axis and the c-axis, respectively. The orientation of the structural unit within the space of the fiber may be specified by using three Euler angles, $\varphi, \theta$ and $\eta$. The angles $\theta$ and $\varphi$, which define the orientation of the $U_{3}$ axis of the unit within the space, are polar and azimuthal angles, respectively, and $\eta$ specifies the rotation of the unit around its own $U_{3}$ axis. For the PBO fibers, the crystal chain axes have a preferential orientation with respect to the $X_{3}$ axis but a random orientation around the $X_{3}$ axis $(\phi$ : random).

The tensor description between the intrinsic compliance of the structural unit and the bulk compliance is given by ${ }^{12-17}$

$$
S_{i j k l}=\sum_{r=1}^{3} \sum_{q=1}^{3} \sum_{p=1}^{3} \sum_{o=1}^{3}\left\langle a_{i o} a_{j p} a_{k q} a_{l r}\right\rangle S_{o p q r}^{c o}
$$

where $S_{i j k l}$ are bulk elastic compliance of the crystal phase and $S_{o p q r}^{c o}$ are their intrinsic compliance. As for a PBO crystal unit, $S_{o p q r}^{c o}$ are given by Tashiro using B-matrix as follows:

$$
S_{u v}^{c o}=\left|\begin{array}{cccccc}
0.1694 & -0.1519 & 0.0001738 & -0.1533 & 0.007022 & -0.4208 \\
-0.1519 & 0.2031 & -0.0006926 & 0.2243 & -0.4969 & 0.4391 \\
0.0001738 & -0.0006926 & 0.001731 & -0.003828 & -0.0002182 & -0.001875 \\
& & & 4.688 & -0.09890 & 0.7310 \\
& & & & 0.3197 & 0.001625 \\
& & & & & 1.490
\end{array}\right|
$$

$a_{i o}$ is, for example, the direction cosine of the $U_{0}$ axis with respect to the $X_{i}$ axis. When the crystallites have a random orientation around the $X_{3}$-axis, average values of the crystal phase in eq 7, $\left\langle a_{i o} a_{j p} a_{k q} a_{l r}\right\rangle_{c v}$, is given by ${ }^{14-19}$

$$
\begin{aligned}
& \left\langle a_{i o} a_{j p} a_{k q} a_{l r}\right\rangle \\
& \quad=\int_{0}^{2 \pi} \int_{0}^{2 \pi} \int_{0}^{\pi} \omega(\theta, \eta) a_{i o} a_{j p} a_{k q} a_{l r} \sin \theta \mathrm{d} \theta \mathrm{d} \phi \mathrm{d} \eta
\end{aligned}
$$

where $\omega(\theta, \eta)$ is the orientation distribution function of the crystal unit $O-U_{1} U_{2} U_{3}$ with respect to the coordinate $O-X_{1} X_{2} X_{3}$ in Figure 7 .

Here it should be noted that the estimation of orientation of PBO crystallites with a triclinic crytsal unit is very complicated as discussed elsewhere for poly(buthylene terephthalate ${ }^{15}$ and poly(ethylene terephthalate ${ }^{16}$ films and nine crystal planes are needed to obtain the fourth order generalized orientation factor for calculating Young's modulus. This evaluation is practically impossible for PBO fibers. Therefore in addition to a random orientation of the structural units around the $X_{3}$-axis (fiber direction), it is assumed that the c-axis has also a random orientation around its own axis. In this case, $\omega(\theta, \eta)$ is independent of $\eta$ and $\omega(\theta)$ can be expanded by the generalized orientation factors $F_{l 00} . F_{l 00}$ can be obtained from the distri-

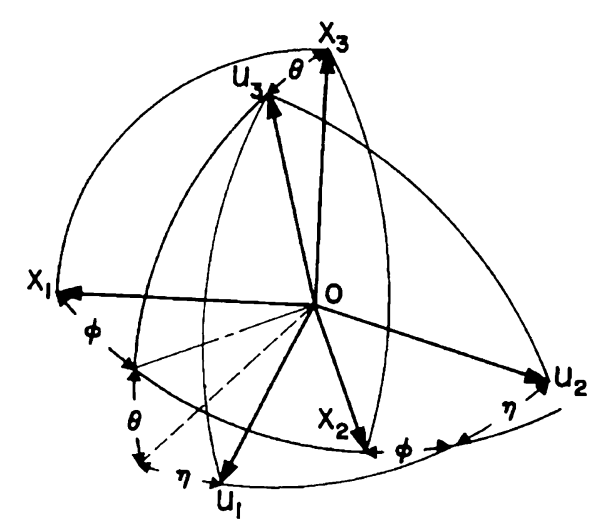

Figure 7. Euler angles $\phi, \theta$ and $\eta$ which specify the orientation of coordinate $O-U_{1} U_{2} U_{3}$ of structural unit with respect to coordinate $O-X_{1} X_{2} X_{3}$ of a specimen. 
bution function of the reciprocal lattice vector of one crystal plane.

In the cylindrical coordinate system in Figure 4, the equation of equilibrium may be obtained as follows:

$$
\frac{\partial \sigma_{r z}}{\partial r}+\frac{1}{r} \frac{\partial \sigma_{\phi z}}{\partial \phi}+\frac{\partial \sigma_{z z}}{\partial z}+\frac{\partial \sigma_{r z}}{r}=0
$$

According to Helmholtz theorem, the displacement components may be expressed in terms of potential function of $\psi$ in the cylindrical coordinate system as follows:

$$
\begin{aligned}
& u_{r}=\frac{\partial \psi}{\partial r}=Q \frac{\partial R}{\partial r} \\
& u_{z}=k_{n} \frac{\partial \psi}{\partial z}=k_{n} \frac{\partial Q}{\partial z} R
\end{aligned}
$$

where

$$
\psi=Q R=\exp (\alpha z)
$$

The coefficient $k_{n}$ in eq 10 is a parameter to provide a suitable solution of eq $9 .^{18,19}$ Substituting eq 4 into eq 9 and then using eq 10, the following equation can be formulated.

$$
\frac{\partial^{2} R}{\partial r^{2}}+\frac{1}{r} \frac{\partial R}{\partial r}+\frac{k_{n} \alpha^{2} C_{33} R}{C_{13}+2 C_{44}\left(1+k_{n}\right)}=0
$$

Eq 12 has a solution represented as

$$
\psi=A J_{0}(\alpha r) \exp (\alpha z)
$$

where $J_{0}(\alpha r)$ is the 0 -th order Bessel function when $k_{n}$ is given by

$$
k_{n}=k=\frac{C_{13}+C_{44}}{C_{33}-2 C_{44}}
$$

When the external stress $T$ is applied along the $X_{3}$ axis, the following boundary conditions must be constructed.

$$
\begin{gathered}
\left.u_{z z}\right|_{\text {in }}=\left.u_{z z}\right|_{\text {out }} \\
\frac{\int_{0}^{a} \sigma_{r r}(\text { in }) r^{2} \mathrm{~d} r}{\int_{a}^{b} \sigma_{r r}(\text { out }) r^{2} \mathrm{~d} r}=\frac{T \pi a^{2}}{T \pi\left(b^{2}-a^{2}\right)}=\frac{1}{\left(b^{2} / a^{2}\right)-1}
\end{gathered}
$$

From the above two conditions, the coefficient $\alpha$ in eq 13 can be determined approximately, and then the stress $\sigma_{r r}$ within the plated fiber can be obtained, if the elastic stiffness $C_{i j}$ of the PBO fiber and the Lame constants of isotropic copper are known. The Lame constants of copper are known parameters but $C_{i j}$ of PBO fibers are unknown. In the present case, the elastic compliances of the amorphous phase are assumed to be equal to those of the crystal phase because of the very rigid properties of a PBO chain. $(b / a)^{2}$ in

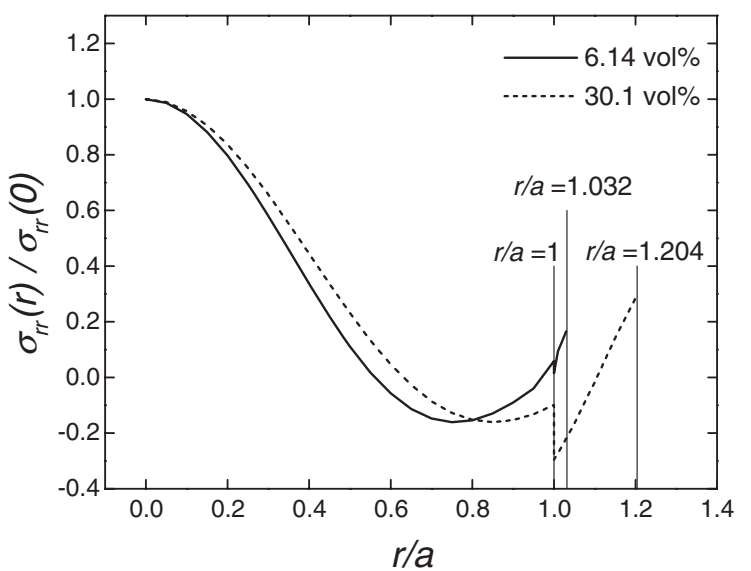

Figure 8. The distribution curves of the normalized stress $\sigma_{r r}(r) / \sigma_{r r}(0)$ in the radial direction as a function of $r / a .1 .032$ and 1.204 are the values of $r / a$ calculated at $\varphi_{V}=6.14$ and $30.1 \mathrm{vol} \%$, respectively, in which $r / a=1$ at the boundary.

eq 16 is given by $1 / \sqrt{\left(1-\varphi_{V}\right)}$.

Figure 8 shows the distribution curves of the stress $\sigma_{r r}$ in the radial direction as a function of $r / a$ normalized at $r=0$, in which $\sigma_{r r}$ is represented as $\sigma_{r r}(r) / \sigma_{r r}(0)$ which is normalized by $\sigma_{r r}$ at $r=0$, because of the difficulty in estimating the real intrinsic stiffness $C_{i j}$ in eq 4. At present, it is impossible to know the values of intrinsic compliance of the amorphous phase as well as the composite mode between the crystal and amorphous phases. In the present paper, we have to use the theoretical values of $C_{i j}$ obtained from the inverse relationship in eq 4 by using theoretical values of $S_{o p q r}^{c o}$ for the crystal phase. Without this assumption, it is impossible to pursue the numerical calculation of the mechanical properties in bulk specimens in relation to crystal units even for usual polymers such as polyethylene ${ }^{20}$ and poly(vinyl alcohol). ${ }^{21}$

The curves were calculated at 6.14 vol \% (27 wt \%) and $30.1 \mathrm{vol} \%(72 \mathrm{wt} \%)$ copper contents, in which the former and latter contents correspond to the values of $r / a, 1.032$ and 1.204 , respectively. The increasing values beyond unity correspond to the thickness of copper layer plated on PBO fiber. At the boundary state between PBO fiber and copper phases, the calculated stress gap occurs and becomes larger with increasing copper content. At $6.14 \mathrm{vol} \%$ content, the gap is very few. The electric conductivity of the plated PBO fiber is $10^{3.9} \mathrm{~S} / \mathrm{cm}$ despite of the small amount of copper plating, and the corresponding Young's modulus becomes $180 \mathrm{GPa}$. Such high values have never been reported for commercial conductive polymers. The conductivity is almost equal to that of bismuth and the Young's modulus is slightly lower than that of nickel. At $30.1 \mathrm{vol} \%$ content, the calculated stress gap at the boundary is much larger than that at 
$6.14 \mathrm{vol} \%$. The electric conductivity of the copperplated PBO fiber is $10^{5.1} \mathrm{~S} / \mathrm{cm}$ corresponding to the conductivity of platinum, and its Young's modulus is about $130 \mathrm{GPa}$, higher than modulus of titanium. The increase in copper content provides an increase in conductivity but a decrease in Young's modulus in comparison with the values of the plated fiber with $6.14 \mathrm{vol} \%$. In spite of the calculated large gap, any peeling phenomenon was not confirmed macroscopically under elongation up to break. This indicates that the tight adhesion at the boundary between PBO and copper phases can conquer the concentrated stress at the boundary. Accordingly, the plating by copper plays an important role to prepare high modulus and high strength fibers with high electric conductivity. However, we must take care of the durability against repeated applied stress along the fiber direction in order to use the plated PBO fibers as electrical wires safely.

\section{CONCLUSION}

Copper plating on the surface of poly ( $p$-phenylenebenzobisoxazole) (PBO) fiber was carried out by using electroless plating. Prior to the plating, the chemical modification of the surface of PBO fibers was done through several processes such as surface coarseness, alkali processing, neutralization, activation and acceleration. The Young's modulus and tensile strength of the plated fiber became lower with increasing copper content but the electric conductivity increased. At the maximum volume fraction of copper, $c a$. $30.1 \mathrm{vol} \%$, the electric conductivity reached $10^{5.1} \mathrm{~S} /$ $\mathrm{cm}$ and the corresponding Young's modulus was $c a$. $130 \mathrm{GPa}$. The stress distribution in the radial direction within the copper-plated fiber was calculated on the basis of continuous mechanical theory. The calculated results indicated that the stress gap at the boundary between PBO and copper phases became larger with increasing copper content. Namely, at $6.14 \mathrm{vol} \%$, the gap was very small, while at 30.1 vol $\%$ content, the gap became very large. In spite of the calculated large gap at $30.1 \mathrm{vol} \%$, any peeling even at $30.1 \mathrm{vol} \%$ content did not occur macroscopically under elongation up to break, indicating that the tight adhesion between the $\mathrm{PBO}$ and copper phases conquers the concentrated stress at the boundary. Accordingly a series of experimental and calculated results suggests future wide utilities of copper-plated PBO fibers as functional fibers.

\section{REFERENCES}

1. T. Kitagawa, H. Murase, and K.Yabuki, J. Polym. Sci., Part B: Polym. Phys., 36, 39 (1998).

2. K. Saito, Y. Takahashi, and M. Sorai, J. Polym. Sci., Part B: Polym. Phys., 38, 1584 (2000).

3. S. J. Krause, T. B. Haddock, D. L. Vezie, P. G. Lenhert, W. F. Hwang, G. E. Price, T. E. Helminiak, J. F. O’Brien, and W. W. Adams, Polymer, 29, 1354 (1988).

4. D. C. Martin and E. L. Thomas, Macromolecules, 24, 2450 (1991).

5. S. Kumar, S. Warner, D. T. Grubb, and W. W. Adams, Polymer, 35, 5408 (1994).

6. M. E. Hunsaker, G. E. Price, and S. J. Bai, Polymer, 33, 2128 (1992).

7. S. J. Bai and G. E. Price, Polymer, 33, 2136 (1992).

8. M. Matsuoka and C. J. Iwakura, J. Electrochem. Soc., 139, 2466 (1992).

9. F. Caturla, F. Molina, M. Molina-Sabio, and J. RodriguezReinoso, J. Electrochem. Soc., 142, 4084 (1995).

10. S. J. Park, Y. S. Jang, and K. Y. Rhee, J. Colloid Interface Sci., 245, 383 (2002).

11. G. M. Wu and Y. T. Shyng, Composites Part A, 35, 1291 (2004).

12. M. Matsuo and C. Sawatari, Macromolecules, 21, 1658 (1988).

13. M. Matsuo, Macromolecules, 23, 3261 (1990).

14. M. Matsuo, C. Sawatari, Y. Iwai, and F. Ozaki, Macromolecules, 23, 3266 (1990).

15. M. Matsuo, R. Adachi, X. Jiang, and Y. Bin, Macromolecules, 37, 1324 (2004).

16. Y. Bin, K. Ooishi, K. Yoshida, T. Nakashima, and M. Matsuo, Polym. J., 36, 394 (2004).

17. Y. Bin, K. Ooishi, K. Yoshida, and M. Matsuo, Polym. J., 36, 888 (2004).

18. M. Matsuo, T. Ogita, S. Suehiro, T. Yamada, and H. Kawai, Macromolecules, 11, 521 (1978).

19. M. Matsuo, J. Chem. Phys., 72, 899 (1980).

20. M. Matsuo and C. Sawatari, Macromolecules, 21, 1653 (1988).

21. M. Matsuo, Y. Bin, and M. Nakano, Polymer, 42, 4687 (2001). 